\title{
Robust Speed Controllers with Autotuning for Electrically Driven Equipment
}

\author{
Andrei Aksjonov \\ ŠKODA AUTO a.s. \\ Mladá Boleslav, Czech Republic \\ andrei.aksjonov@skoda-auto.cz
}

\author{
Aleksandr Serbin \\ ABB a.s. \\ Tallinn, Estonia \\ aleksandr.serbin@gmail.com
}

\author{
Valery Vodovozov and Zoja Raud \\ Tallinn University of Technology \\ Tallinn, Estonia \\ valery.vodovozov@taltech.ee
}

\begin{abstract}
In the light of the growing demands for robustness of machine tools and apparatus equipment, this paper focuses on the speed control of electrically driven moving parts, such as supports, cars, loaders, forklift trucks, carriers, etc. The research lays emphasis on how to maintain identical dynamics in terms of the speed overshoot and the speed rise time at driving obstacles that cannot be confidently foreseen. This issue is typical for machines operated in rather stable conditions at moderate speeds those frictions, gaps, backlashes, or elasticity may change unpredictably during operation. Two autotuning procedures are designed and studied. The first one based on an iterative binary logic algorithm addresses the accurate following the demanded dynamics. The second one based on the one-step fuzzy logic approach provides rapid achieving the setpoint with rather good dynamics.
\end{abstract}

Keywords-electrical drive, machine tools, PID controller, binary logic controller, fuzzy logic controller, autotuning.

\section{INTRODUCTION}

Electrically driven equipment is frequently among the complex nonlinear and non-stationary applications running in different modes, such as rapid move with acceleration and slowing down, heavy braking, and positioning. Every mode calls its specific requirements with regard to dynamic performance, steady-state accuracy, speed range, torque stability, and overload capacity in the face of changing load circumstances. To prevent vague and unpredictable behaviour, the control system places high demands on intelligent controllers that would satisfy both offline manual tuning and online autotuning capable to make decisions without human intervention, if need be.

Oftentimes, a conventional proportional-integralderivative controller (PIDC) is employed, which works properly under a specific set of known system parameters and load conditions. Tuning process of the PIDC is quite elaborated for both the offline and the online stages. Offline tuning is based on the simplified mathematical model of the controlled plant and disturbances, their gains and time constants, to name a few. Because of proliferation of uncertainties, not all data can be exactly known offline; therefore, a natural step forward along the line is to consider autotuning aiming to improve PIDC settings online to meet the real-time needs. Online tuning is applied in practice based on critical points and characteristics of the plant using typical drive reactions, including step response, frequency response, closed-loop relay feedback, etc. Very popular are well-known tuning methods, such as Ziegler-Nichols, Cohen-Coon, Chien-Hrones-Reswick, or more sophisticated schemes [1] that provide different types of loop performance. This technique guarantees enough accurate tuning for stable modes of equipment running when the setpoint changes larger and faster than the control variables while disturbances appear as slow departures of control variables from the setpoint. However, many occasions prevent expansion of the PIDC in electric motion control:

- in unstable and changing conditions, the settings that produce a desired response at one operating point usually do not call a satisfactory response in other circumstances [2];

- PIDC operates perfectly at small deviations of the controlled variables, whereas considerable speed or torque modification call the control loops to saturate with feedbacks disconnection [3];

- PIDC is designed to work in the systems with single input and single output, but the circuits with multiple inputs and single (MISO) or multiple (MIMO) outputs often fail in acceptable performance [4]

To resolve these conflicting issues, the gain scheduling method is somewhere applied [5], [6], which helps in choosing optimal PIDC settings that best satisfy the current range of plant variables. Nevertheless, gain scheduling cannot consider all possible system states to ensure overall robustness.

In that sense, designers of the robust systems force to apply to the fuzzy logic controller (FLC) [7] that, in contrast to classical binary logic, deals with fuzzy sets of linguistic variables (LV) capable to partial membership between 0 (absence of membership) and 1 (full membership) rather than to crisp membership (0 or 1$)$. Thanks to its membership function (MF), the FLC is able to govern nonlinear and complex plants that are difficult to characterise mathematically but may be described qualitatively. However, despite the numerous FLC benefits, it is worthy of note FLC weakness in terms of the accurate motion control:

- in contrast to PIDC, the FLC has no internal dynamic aspects, i.e. its input/output characteristic fields have usually a stepwise pattern [8] unsuitable for speed adjustment, acceleration, or slowing down;

- attempting to develop a MIMO FLC, such as in [9], is usually daunting as it is difficult to establish fuzzy relations between significant number of variables based primarily on human expertise and skills;

- although new sliding and adaptive approaches alleviate to a certain extent the difficulties in constructing fuzzy rule bases [10], they, just like the traditional trial-and-error methods, remain quite sensitive to practitioners' cognitive biases that hamper control reliability. 
As a result, most of often-cited FLCs are valid only within the specific bands of parameters and variables. This is a severe restriction on general implementation of FLCs since they require extensive retuning [11] - [14].

Starting with [8], [15], it has been found that the fuzzy PIDC has better handling capabilities than both the PIDC and the FLC separately as it preserves the simple linear principle of PIDC operation. Nonetheless, such negative issue of the fuzzy PIDC as difficulty in MIMO arrangement complicates construction and autotuning of fuzzy PIDCs. There are rather few publications about fuzzy PIDCs capable to convert at least two inputs (usually, the speed error and its rate) directly into at least three PIDC settings [9], [16] - [18]. A more prevalent approach presented, in particular, in [6], [19] - [21] involves sharing of fuzzy operation among three independent controllers, namely, fuzzy $\mathrm{P}$, fuzzy $\mathrm{I}$, and fuzzy D.

Aside from direct problem solving, an essential idea of many fuzzy PIDC algorithms lies in the MIMO system alignment with the MISO one by converting the error signal (speed) and its time derivative (acceleration) into some aggregated output with a linear-like control surface [22], which actually combines fuzzy PI and fuzzy PD controllers. Particularly, in [15] a Ziegler-Nichols formula is parameterized by one common variable. Through the fuzzy PIDCs often fail in using Mamdani's-type reasoning [23], the two-term fuzzy controllers, PI and PD [10], PD and I [18] or PD and ID [11] may be successfully realised. However, the problem of optimal tuning for all PIDC parts remains open in these systems because their control actions are strongly coupled. The contribution of scaling gains to the output action remains unclear that makes known tuning methodologies rather unreliable.

To address the challenges of nonlinearity and timevariability, nonlinear control terms were proposed in [4], [17] and [24], but these techniques are also limited by the fixed range of parameters, resulting in frequent detuning to accommodate worst-case scenarios, such as the motion upon bad lubrication with uneven guiding skids, for instance.

Unlike the above listed works and based on the authors' previous FLC designs [25], [26], the present research lays emphasis on how to maintain maybe not the best on its own, but some optimal (say, sample) dynamics in terms of the speed overshoot and rise time at changeable moving modes and loading conditions. To that end, the paper addresses two aspects, namely, accurate following the demanded dynamics on the one hand and rapid achieving the setpoint with rather afforable dynamics on the other. This issue is typical for machines operated in more or less stable conditions at moderate speeds those frictions, gaps, backlashes, or elasticity may change unpredictably during operation. The study focuses on the PIDC autotuning in the MIMO system with two input signals, the slope error, and the peak error, by means of the binary logic and the fuzzy logic approaches. LabVIEW $^{\mathrm{TM}}$ toolkit from National Instruments ${ }^{\circledR}$ is used in this research as a convenient simulation tool and an intuitive graphical programming user interface for data acquisition and analysis of drives with PIDC, FLC, and fuzzy PIDC.

The paper is organised as follows. First, the principles are justified for a versatile controller composition equally suitable for speed adjustment, acceleration, and slowing down under different conditions. Next, controller tuning and binary logic autotuning peculiarities are explained with integration in the control system. Then, the designed fuzzy PIDC is demonstrated and the conclusions are drawn.

\section{SPEED CONTROL SYSTEM}

To design a controller, the control variables $y$, setpoints $y^{*}$, and control demands $x$ have to be specified (Fig. 1, a) as well as the crisp range of all their possible values called a universe of discourse (UOD). Every mode of machine running has its specific control variables: speed for motion, position for orientation, torque for braking, etc. These variables may be regulated in serial cascades, in parallel, or in hybrid combinations.

In this study, arrangement of the speed controller is discussed, which inputs are the setpoint speed $\omega^{*}$, the control variable - real plant speed $\omega$, and the output $x$ is the speed control demand. A linear model shown in Fig. 1, b is used as the first approximation. Here, $s$ is the Laplace operator, $\delta=$ $\omega^{*}-\omega-$ speed error, $E-$ motor electromotive force representing the control demand $x, T_{p}-$ plant torque representing the main disturbance, $W_{c}(s)$ - transfer function of the designed controller, and $W_{p}(s)$ - transfer function of the controlled plant. In the closed loop assumed by means of the transfer function

$$
W(s)=\frac{\omega(s)}{\omega^{*}(s)}=\frac{W_{c}(s) W_{p}(s)}{1+W_{c}(s) W_{p}(s)}
$$

the transfer function of the controller is as follows:

$$
W_{c}(s)=\frac{1}{W_{p}(s)} \cdot \frac{W(s)}{1-W(s)} .
$$

This image includes the transfer function of the plant and a part, which depends on the desired system model, $W(s)$. Usually, transfer functions $W(s)$ differ depending on their characteristic polynomials and on the process dead time $\tau_{\mu}$. To enable equally balanced speed performance for both disturbance rejection and reference signal tracking, the transfer function presented by the second-order characteristic polynomial

$$
a_{1} \tau_{\mu}^{2} s^{2}+a_{1} \tau_{\mu} s+1
$$

best fulfils when $2 \leq a_{1}<4$. At that, the plant has the nonperiodic step response with the small overshoot [27] used here as a sample response towards the given dead time.

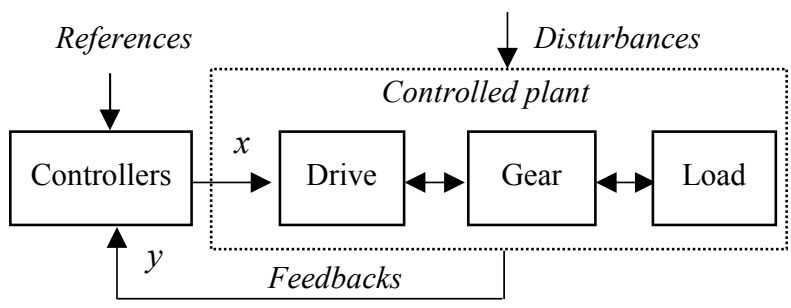

a.

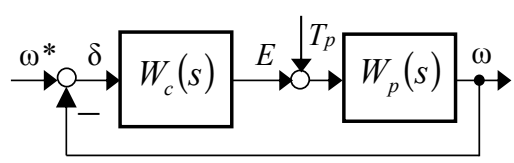

b.

Fig. 1. Functional diagram of the control system (a) and its approximated linear model (b). 
With regard to the approximated plant model, the following pair of the Laplace's equations describes the speed responses on the step reference and disturbance:

$$
\begin{aligned}
& \omega(s)=E(s) \frac{k}{\tau \tau_{\mu} s^{2}+\tau s+1}, \\
& \omega(s)=-T_{p}(s) \frac{\tau\left(\tau_{\mu} s+1\right)}{J\left(\tau \tau_{\mu} s^{2}+\tau s+1\right)} .
\end{aligned}
$$

Here, $k=\frac{I}{T}$ is the motor gain expressed by the ratio between its passport current $I$ and torque $T ; T_{p}$ - plant torque represented the main disturbance; $\tau=\frac{J \omega_{\max }}{T_{\max }}-$ plant time constant; $J$ - its moment of inertia; $\omega_{\max }, T_{\max }-$ maximal speed and torque of the motor.

Once the desired system and the plant models are defined, the PIDC may be tuned, which transfer function in the operator domain is as follows:

$$
W_{c}(s)=\frac{E(s)}{\delta(s)}=k_{c}\left(1+\frac{1}{\tau_{i n t} s}+\tau_{d i f} s\right) .
$$

Here, $k_{c}$ is the controller gain that defines the regulation accuracy, $\tau_{i n t}$ - integral time constant that forces the steadystate error to zero, and $\tau_{d i f}$ - derivative time constant that accelerates dynamics whenever necessary. The output of the PIDC fitted to its UOD $\left\{x_{\min } ; x_{\max }\right\}$ is applied as a control demand $E$ to the drive aiming to shift the control variable $\omega$ to the setpoint $\omega^{*}$ for minimising the speed error $\delta$.

\section{PIDC TUNING AND BINARY LOGIC AUtOTUNING}

At first, the PIDC is tuned offline to obtain the required steady-state accuracy and sample dynamics exemplified in Fig. 2 by the solid line. Proven decision-making rules used for tuning the proportional, integral, and derivative parts of the PIDC are given, particularly, in [27].

As offline tuning is based on approximated linear plant models, the issues associated with system nonlinearity and load instability usually lead to the loop behaviour distortion. This performance deterioration is referred to as windup, which may further result in big jumps in the plant input and poor tracking performance, referred to as bump transfer [28]. Often, the system appears either too sluggish (dashed lines in Fig. 2) or too aggressive (dotted lines) comparing with the sample response chosen as a design target. Against this backdrop, an online autotuning is initialised.

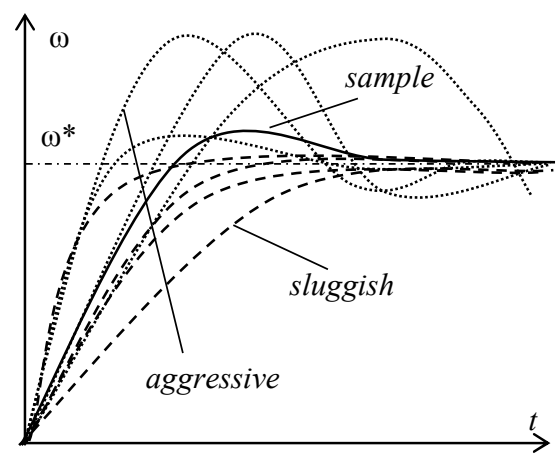

Fig. 2. Step responses and distortion combinations.
To that end, the controller periodically evaluates the trend of the plant response aiming to detect its deviation from a prescribed sample course. If a deviation is found, a control action is generated to correct the PIDC settings with the following essence.

Every abnormal response is seen as a combination of two types of dynamic errors: the slope and the peak.

The slope characterises the speed rise time, i.e. the firstmatching time during which the speed response approaches the setpoint. Let the slope error is the ratio between the rise time $t_{\left(\omega=\omega^{*}\right)}$ of the sample speed response and the rise time of the distorted process:

$$
\text { slope }=\frac{t_{\text {sample }\left(\omega=\omega^{*}\right)}}{t_{\operatorname{proc}\left(\omega=\omega^{*}\right)}} .
$$

The peak characterises the speed overshoot. Let the peak error is the ratio between the overshoot $\delta_{(\max )}$ of the distorted process and the overshoot of the sample one:

$$
\text { peak }=\frac{\delta_{\text {proc }(\max )}}{\delta_{\text {sample }(\max )}} .
$$

Based on the above definitions, the following stepwise binary logic PIDC autotuning algorithm is implemented:

- to reduce (or enlarge) a peak, the time constant $\tau_{\text {int }}$ is increased (or, appropriately, decreased) in increments, and, if possible, the time constant $\tau_{\text {dif }}$ is decreased (or increased), until the sample peak reaching;

- as any change in $\tau_{i n t}$ courses the reverse change of the slope, the slope correction is usually further required;

- to reduce (or enlarge) a slope, the gain $k_{c}$ is decreased (or, appropriately, increased) in increments, until the sample slope reaching.

The simple-iteration method with alternating step size was employed for the gain and time constants reduction and enlargement. The step size is defined by the size of error whereas the number of steps depends on the slope and peak tolerances.

It is noteworthy that the derivative term is seldom employed, especially in speed control, mainly due to the fact, that it increases sensibility to noise. Although most of the PIDCs incorporate this action, it is quite usual for the plant operators to inhibit it. The derivative mode may be used to speed up running or braking whenever the large error appears, whereas the integral mode is commonly applied in small error conditions. Thereby, the derivative term is accompanied here by "if possible" remark.

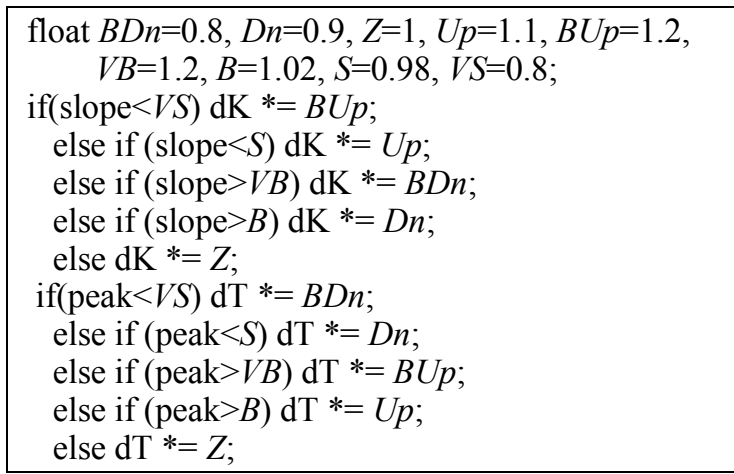

Fig. 3. LabVIEW code of the binary logic autotuning algorithm. 


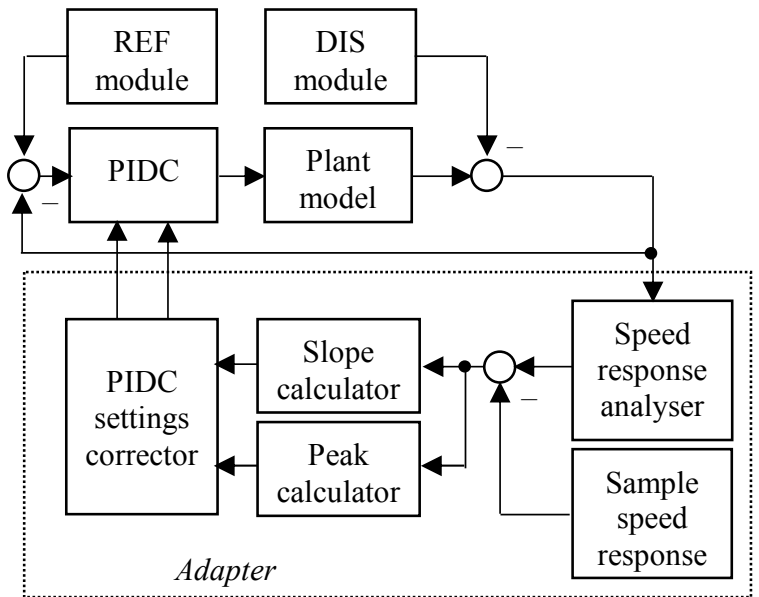

Fig. 4. Model of the speed control system with autotuning.

In Fig. 3, the LabVIEW code of the algorithm used for the PI controller autotuning is presented. Its input variables are the current slope and peak values, whereas the output variables define the signals $\mathrm{dK}$ used for the gain correction and $\mathrm{dT}$ for the integral time constant correction. Error and step crisp sizes at $\pm 2 \%$ tolerance are announced in the code header. "If-Then" operators process these variables using binary logic. The algorithm is running periodically thus enabling iterative functionality for autotuning.

In Fig. 4, the model of the speed control system with autotuning is shown. Here, the reference (REF) and disturbance (DIS) modules generate the PIDC and plant speed responses directed to the adapter, which compares the plant response with the sample one aiming to normalize the slope and peak errors. The PIDC settings corrector employs the above code to generate correction signals.

In Fig. 5, the designed LabVIEW user interface is presented. Here, two selectors are intended to choose between the simulation modes: the Reference/Disturbance switch and the Manual/Auto switch. REF settings, DIS settings, Manual PID settings, and PID autotuning settings occupy the separate areas alongside the response chart display. A line of placards reflects process information.

The main benefit of this binary logic autotuning procedure is its accuracy whereas the drawbacks concern its slowness.

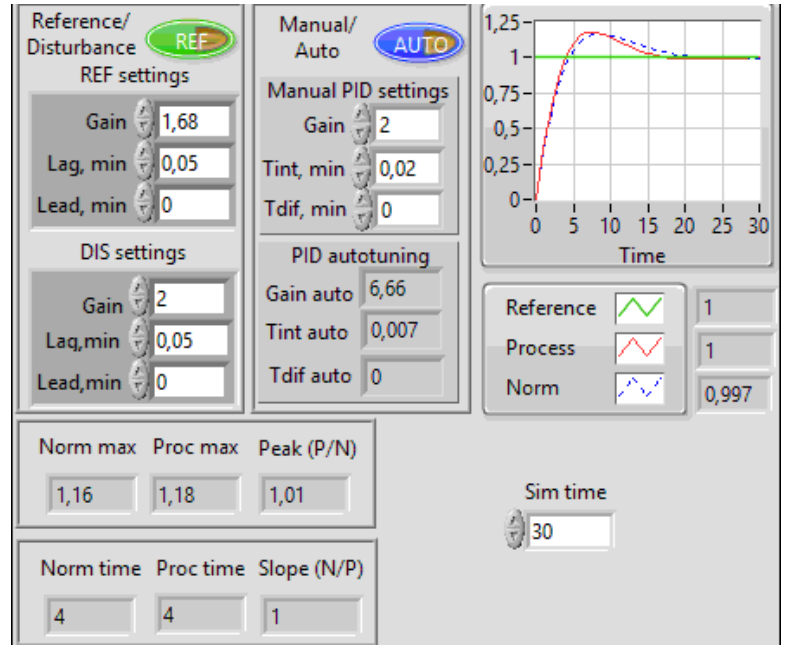

Fig. 5. LabVIEW user interface of the speed control system.
The simulation shows that it costs a long time to settle for a step response, especially in large inertia delay systems. The adapter sometimes requires up to 50 online PIDC cycles for full settings autotuning.

\section{SPEEd PIDC with FuZZy Logic AUtotuning}

As it was proven in [29], non-linearly constrained and badly modelled problems can successfully be solved using fuzzy approach in lesser number of iterations because, due to the limits on the feasible area, rather "clever" adapter can make well-informed decisions regarding directions of search and step size. Therefore, to speed up the process, the binary logic adapter may be replaced with the FLC.

The designed speed fuzzy PI controller has a MIMO topology with the Mamdani's-type inference mechanism. The slope and the peak errors were assigned as input LVs (Table I). From the slope side, the responses are classified using the following fuzzy sets: $V B$ - very bluff $(>>1), B-$ bluff ( $>1), N-$ normal (within the tolerance range), $S-$ sloping $(<1)$, and $V S$ - very sloping $(<<1)$. Depending on the peak errors, the responses are classified as $V B$ - very big ( $>>1), B-$ big $(>1), N-$ normal (within the tolerance range), $S-$ small $(<1)$, and $V S$ - very small $(<<1)$. The PIDC gain correction $\mathrm{dK}$ and the PIDC integral time constant correction dT were chosen as output LVs those MFs were assigned as $B D n$ - big down, $D n$ - down, $Z$ - zero change, $U p$ - up, and $B U p$ - big up.

TABLE I. RULE BASE OF SPEED FLC

\begin{tabular}{|c|l|l|l|l|l|}
\hline \multirow{2}{*}{ slope } & \multicolumn{5}{|c|}{ peak } \\
\cline { 2 - 6 } & \multicolumn{1}{|c|}{$\boldsymbol{V} \boldsymbol{S}$} & \multicolumn{1}{|c|}{$\boldsymbol{S}$} & \multicolumn{1}{|c|}{$\boldsymbol{\boldsymbol { B }}$} & \multicolumn{1}{c|}{$\boldsymbol{B}$} \\
\hline \multirow{3}{*}{$\boldsymbol{V} \boldsymbol{S}$} & $\mathrm{dK}=B U p$ & $\mathrm{dK}=B U p$ & $\mathrm{dK}=B U p$ & $\mathrm{dK}=B U p$ & $\mathrm{dK}=B U p$ \\
\cline { 2 - 6 } & $\mathrm{dT}=B D n$ & $\mathrm{dT}=D n$ & $\mathrm{dT}=Z$ & $\mathrm{dT}=U p$ & $\mathrm{dT}=B U p$ \\
\hline \multirow{2}{*}{$\boldsymbol{S}$} & $\mathrm{dK}=U p$ & $\mathrm{dK}=U p$ & $\mathrm{dK}=U p$ & $\mathrm{dK}=U p$ & $\mathrm{dK}=U p$ \\
\cline { 2 - 6 } & $\mathrm{dT}=B D n$ & $\mathrm{dT}=D n$ & $\mathrm{dT}=Z$ & $\mathrm{dT}=U p$ & $\mathrm{dT}=B U p$ \\
\hline \multirow{2}{*}{$\boldsymbol{N}$} & $\mathrm{dK}=Z$ & $\mathrm{dK}=Z$ & $\mathrm{dK}=Z$ & $\mathrm{dK}=Z$ & $\mathrm{dK}=Z$ \\
\cline { 2 - 6 } & $\mathrm{dT}=B D n$ & $\mathrm{dT}=D n$ & $\mathrm{dT}=Z$ & $\mathrm{dT}=U p$ & $\mathrm{dT}=B U p$ \\
\hline \multirow{2}{*}{$\boldsymbol{B}$} & $\mathrm{dK}=\mathrm{Dn}$ & $\mathrm{dK}=D n$ & $\mathrm{dK}=D n$ & $\mathrm{dK}=D n$ & $\mathrm{dK}=D n$ \\
\cline { 2 - 6 } & $\mathrm{dT}=B D n$ & $\mathrm{dT}=D n$ & $\mathrm{dT}=Z$ & $\mathrm{dT}=U p$ & $\mathrm{dT}=B U p$ \\
\hline \multirow{2}{*}{$\boldsymbol{V} \boldsymbol{B}$} & $\mathrm{dK}=B D n$ & $\mathrm{dK}=B D n$ & $\mathrm{dK}=B D n$ & $\mathrm{dK}=B D n$ & $\mathrm{dK}=B D n$ \\
\cline { 2 - 6 } & $\mathrm{dT}=B D n$ & $\mathrm{dT}=D n$ & $\mathrm{dT}=Z$ & $\mathrm{dT}=U p$ & $\mathrm{dT}=B U p$ \\
\hline
\end{tabular}
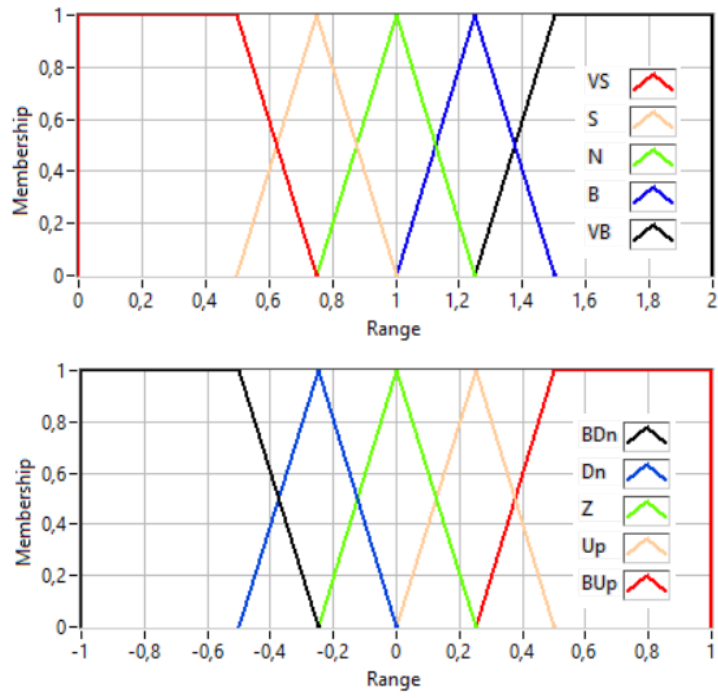

Fig. 6. Fuzzy sets for the input and output LVs. 


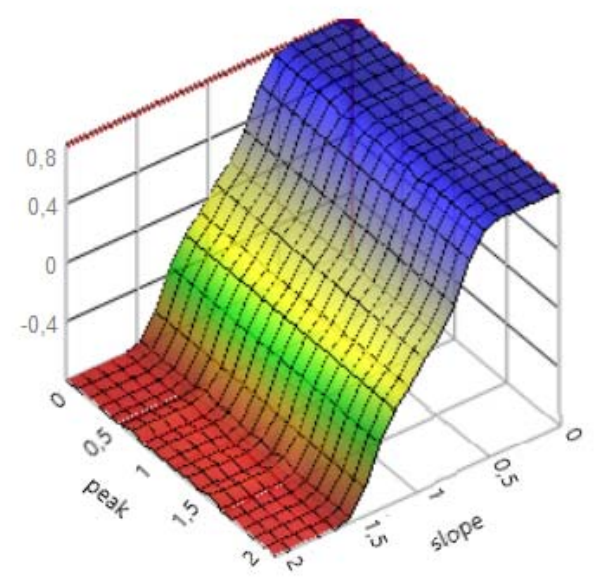

Fig. 7. One of the three-dimensional surfaces.

By applying "If-Then" modus ponens, an appropriate rule base has been developed. Using the centre of gravity as a defuzzification method, two outputs are further converted to the enhanced crisp settings: $k_{c}$ and $\tau_{\text {int }}$. Fig. 6 represents the fuzzy triangle sets for the input and output MFs that have closed frontiers of UODs.

One of the three-dimensional surfaces of these fuzzy sets is shown in Fig. 7.

\section{EXPERIMENTAL VALIDATION}

To validate the designed controllers, the settings obtained from their autotuning procedures were uploaded to the PI controller of the laboratory setup FESTO ${ }^{\circledR}$ TP801 equipped with a brushless dc motor MTR-AC55, a servo converter SEC-AC305, and an axis slider replicated a moving machine part. The plant has the following data: nominal motor voltage $-325 \mathrm{~V}$, current $-2.15 \mathrm{~A}$, torque $-0.66 \mathrm{Nm}$, and speed $6800 \mathrm{rpm}$; maximal current $-6.4 \mathrm{~A}$ at torque $-0.98 \mathrm{Nm}$; moment of inertia of the drive $-1 \mathrm{kgcm}^{2}$ and dead time $15 \mu \mathrm{s}$.

Experimental speed and current traces of a detuned (a), configured with the binary logic controller settings (b), and configured with the FLC settings (c) are shown in Fig. 8. Experimentation demonstrates that the PI controller settings obtained from the binary logic algorithm provides the most accurate slider run up with sample speed overshoot whereas the FLC-driven process looks rather sluggish but enough accurate as well.

\section{CONCLUSIONS}

The proposed PIDCs with autotuning have competitive advantages over the separate PIDC and FLC. Their slope and peak input signals are used for setting and correcting the gain and time constants. System tuning can be conducted partly offline and partly directly in the automated process by analysing the speed responses. Both the binary logic and the fuzzy logic controllers are capable to implement nonlinear control strategies described by the mathematical and the linguistic variables, appropriately, in the conditions when the process and the plant models change unpredictably. An iterative binary logic algorithm addresses the accurate following the demanded dynamics. The one-step fuzzy logic approach provides rapid achieving the setpoint with rather good dynamics. The offered approach has high versatility as it supports different plant running modes.
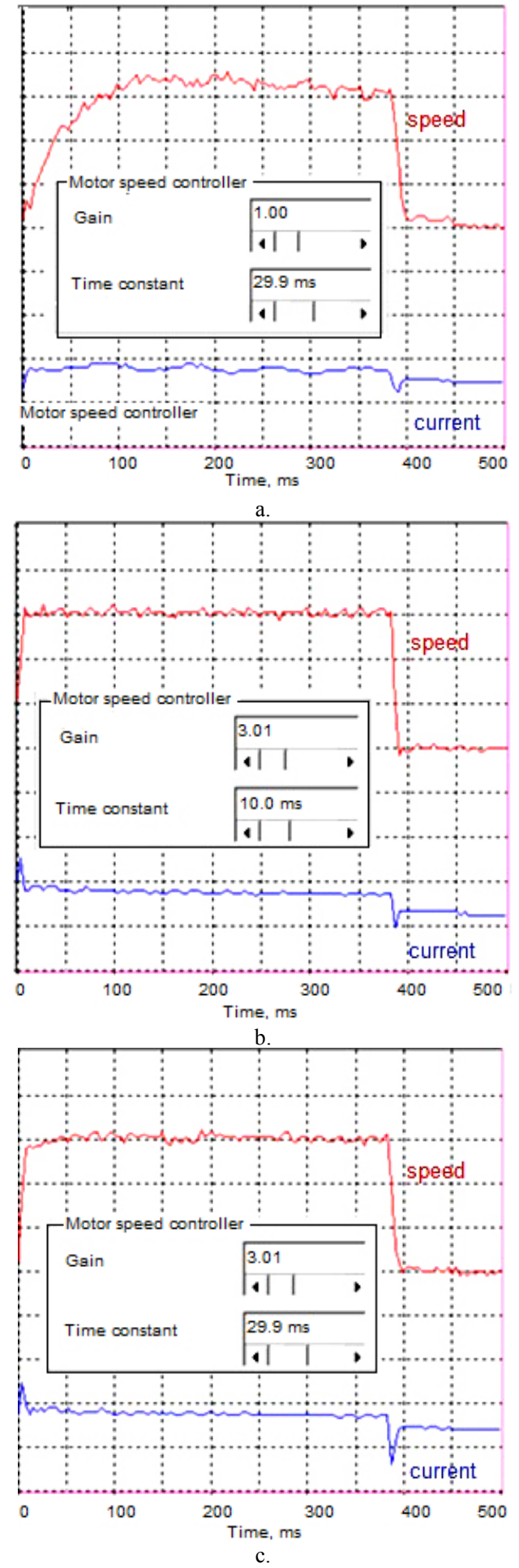

Fig. 8. Experimental traces recorded from the laboratory setup.

\section{ACKNOWLEDGEMENT}

This study has received partial funding from the European Union's Horizon 2020 research and innovation program under grant agreement No. 675999.

\section{REFERENCES}

1 M. Korkmaz, Ö. Ydoğdu and H. Doğan, "Design and performance comparison of variable parameter nonlinear PID controller and genetic algorithm based PID controller," International Symposium on Innovations in Intelligent Systems and Applications, Trabzon, Turkey, 2012, pp. $1-5$. 
2 H. Zhou, F. Jia, H. Jing, Z. Liu and L. Güvenç, "Coordinated longitudinal and lateral motion control for four wheel independent motor-drive electric vehicle," IEEE Transactions on Vehicular Technology, vol. 67, no. 5, pp. 3782 - 3790, May 2018.

3 J. Zhao, Y. Ma, H. Zhao, Y. Cui and H. Chen "PID slip control based on vertical suspension system for in-wheel-motored electric vehicles," Chinese Control and Decision Conference (CCDC), Shenyang, China, 2018, pp. $1126-1131$.

4 P. Gil, C. Lucena, A. Cardoso and L. B. Palma, "Gain tuning of fuzzy PID controllers for MIMO systems: A performance-driven approach," IEEE Transactions on Fuzzy Systems, vol. 23, no. 4, pp. 757 - 768, Aug. 2015.

5 LabVIEW PID and Fuzzy Logic Toolkit User Manual, National Instruments, 2009, $126 \mathrm{p}$.

6 A. M. O. Fini, M. B. Gogani and M. Pourgholi, "Fuzzy gain scheduling of PID controller implemented on real time level control," 4th Iranian Joint Congress on Fuzzy and Intelligent Systems (CFIS), Zahedan, Iran, 2015, pp. $1-5$.

7 K. S. Jena, A. V. Joseph and P. R. R. Senapati, "Fuzzy logic based approach for controlling of a vehicle in its longitudinal motion, Middle-East Journal of Scientific Research, vol. 24 (S1), pp. 346 352, 2016.

8 W. Siler and H. Ying, "Fuzzy control theory: The linear case," Fuzzy Sets Syst., vol. 33, pp. 275 - 290, 1989.

9 P. Xue, H. Wang, J. Hou and W. Li, "Based on the fuzzy PID brushless DC motor control system design," International Conference on Measurement, Information and Control (MIC), Harbin, China, 2012, pp. $703-706$.

10 B. M. Mohan and A. Sinha, "Analytical structures for fuzzy PID controllers?," IEEE Transactions on Fuzzy Systems, vol. 16, no. 1, pp. $52-60$, Feb. 2008 .

11 Q. Li and D. Shen, "A new incremental fuzzy PD+fuzzy ID fuzzy controller," WASE International Conference on Information Engineering, Taiyuan, China, 2009, pp. $615-619$.

12 M. Santos, S. Dormido and J. M. de la Cruz, "Fuzzy PID convrollers vs. fuzzy PI controllers," 5th IEEE International Conference on Fuzzy Systems, New Orleans, LA, USA, 1996, pp. 1598 - 1604.

13 J. Soriano, A. Olarte and M. Melgarejo, "Fuzzy controller for MIMO systems using defuzzification based on boolean relations (DBR)," 14th IEEE International Conference on Fuzzy Systems, Reno, NV, USA, 2005 , pp. $271-275$.

14 Y. Jian and L. Changliang, "Design of self-tuning PID controller with fuzzy variable parameters based on LabVIEW," IEEE International Conference on Information and Automation, Lijiang, China, 2015, pp. $2586-2591$

15 S. Z. He, S. H. Tan, F. L. Xu and P. Z. Wang, "PID self-tuning control using a fuzzy adaptive mechanism," 2nd IEEE International Conference on Fuzzy Systems, San Francisco, CA, USA, 1993, pp. $708-713$.

16 Y. Xiaojin, P. Yan, S. Jinhao, L. Yezi and Q. Jianling, "Self-adaptive tuning of fuzzy PID control of PV grid-connected inverter," 6th
International Conference on Fuzzy Systems and Knowledge Discovery, Tianjin, China, 2009, pp. 160 - 162.

17 L. P. Sun, Z. H. Li and W. H. Cao, "Design and realization of fuzzy self-tuning PID controller based on TMS320LF2407A DSPs for microwave-vacuum wood drying," 6th International Conference on Fuzzy Systems and Knowledge Discovery, Tianjin, China, 2009, pp. $71-75$.

18 A. F. Amer, E. A. Sallam and W. M. Elawady, "Fuzzy precompensated fuzzy self-tuning fuzzy PID controller of 3 DOF planar robot manipulators," IEEE/ASME International Conference on Advanced Intelligent Mechatronics, Montreal, Canada, 2010, pp. 599 604.

19 A. Rubaai, M. J. Castro-Sitiriche and A. Ofoli, "DSP-based implementation of fuzzy-PID controller using genetic optimization for high performance motor drives," IEEE Industry Applications Annual Meeting, New Orleans, LA, USA, 2007, pp. 1649 - 1656.

20 S. H. Lian, C. H. Messom and Y.P. Kong, "Fuzzy hybrid PID controller of a steam heated dryer," IEEE International Fuzzy Systems Conference, Seoul, South Korea, 1999, pp. 1707 - 1711.

21 K. Sharma and D. K. Palwalia, "A modified PID control with adaptive fuzzy controller applied to DC motor," International Conference on Information, Communication, Instrumentation and Control (ICICIC), Cairo, Egypt, 2017, pp. $1-6$.

22 B. Hu, G. K. I. Mann and R. G. Gosine "New methodology for analytical and optimal design of fuzzy PID controllers," IEEE Transactions on Fuzzy Systems, vol. 7, no. 5, pp. 521 - 539, Oct. 1999.

23 M. Mizumoto, "Realization of PID controls by fuzzy control methods," IEEE International Conference on Fuzzy Systems, Adelaide, Australia, 1992 , pp. $709-715$.

24 X.-G. Duan, H.-X. Li and H. Deng "A simple tuning method for fuzzy PID control," IEEE International Conference on Fuzzy Systems, Hong Kong, China, 2008, pp. $271-275$.

25 A. Aksjonov, V. Vodovozov and E. Petlenkov, "Design and experimentation of fuzzy logic control for an anti-lock braking system," 15th Biennial Baltic Electronics Conference (BEC), Tallinn, Estonia, 2016, pp. $207-210$.

26 A. Aksjonov, V. Vodovozov, K. Augsburg and E. Petlenkov, "Design of regenerative anti-lock braking system controller for 4 in-wheelmotor drive electric vehicle with road surface estimation," International Journal of Automotive Technology 19 (4), pp. 727 - 742, 2018.

27 V. Vodovozov, Electrical Drive: Performance, Design and Control, Saarbrücken, Germany: LAP, 2014, 320 p.

28 Y. Peng, D. Vrancic, and R. Hanus, "Anti-windup, bumpless, and conditioned transfer techniques for PID controllers," IEEE Control Systems Magazine, vol. 16 , no. 4, pp 48 - 57, Aug. 1996.

29 Y. Huang and S. Yasunobu, "A general practical design method for fuzzy PID control from conventional PID control," 9th IEEE International Conference on Fuzzy Systems, San Antonio, TX, USA, 2000, pp. $969-972$. 